\title{
Memories of reward events and expectancies of reward events may work in tandem
}

\author{
E. J. CAPALDI, KIMBERLY M. BIRMINGHAM, and SUZAN ALPTEKIN \\ Purdue University, West Lafayette, Indiana
}

\begin{abstract}
A common assumption is that expectancies of reward events in instrumental tasks are established on the basis of Pavlovian conditioning. According to the tandem hypothesis, tested in the four runway investigations reported here employing rats, memories of reward events may serve as the conditioned stimuli eliciting expectancies. In Experiments 1-3, rats were trained under a schedule of partial reward (P), which did not produce increased resistance to extinction, and subseguently shifted to consistent reward (C). According to the tandem hypothesis, the shift to the $\mathrm{C}$ schedule should result in increased resistance to extinction if, as hypothesized, under the P schedule the memory of reward, $\mathrm{S}^{\mathrm{R}}$, came to elicit the expectancy of nonreward, $\mathrm{E}_{\mathrm{N}}$. This hypothesis was confirmed under a variety of conditions. It was shown that increased resistance to extinction could not be attributed to the $\mathrm{P}$ schedule alone, to the rats receiving two schedules, $\mathrm{P}$ and $\mathrm{C}$, to stimuli other than $\mathrm{S}^{\mathrm{R}}$ eliciting $\mathrm{E}_{\mathrm{N}}$, or to the rats forgetting reward-produced memories when expecting nonreward (Experiment 4). It was shown that the tandem hypothesis could explain the divergent findings obtained in prior studies employing a shift from $\mathrm{P}$ to $\mathrm{C}$ as well as in the present study.
\end{abstract}

In Pavlovian conditioning, as Hilgard and Marquis (1940) have indicated, "any environmental change to which the organism is sensitive may serve as the conditioned stimulus" (p. 35). As temporal conditioning illustrates, even an internal stimulus may serve as the conditioned stimulus. The four investigations reported here were concerned with determining whether a particular class of internal stimuli, reward-produced memories, might serve as conditioned stimuli.

Substantial evidence indicates that two sorts of representations of reward events may control instrumental responding: the expectancy of reward events contingent upon the current response, and the memory of reward events contingent upon one or more prior responses. In instrumental tasks, it is widely regarded that expectancies are established on the basis of Pavlovian conditioning (e.g., Amsel, 1958, 1992; Tolman, 1934). Among the conditioned stimuli giving rise to expectancies may be either exteroceptive cues, such as tones or lights, or rewardproduced memories (Capaldi, 1994). Which of the two classes of stimuli mentioned above becomes the stronger signal for expectancy would depend upon their relative validities, and in some, and perhaps many, instrumental situations, the reward-produced memories are more valid than exteroceptive cues and, indeed, may be at least as salient (see, e.g., Haddad, Walkenbach, Preston, \& Strong, 1981; Haggbloom, 1981).

E. J. Capaldi's mailing address is Department of Psychology, 1364 Psychology Building, Purdue University, West Lafayette, IN $47907-$ 1364 (e-mail: julie@psych.purdue.edu).

-Accepted by previous editor, Vincent M. LoLordo
Reward-produced memories may be relatively more valid than exteroceptive cues because rats are capable of remembering whether one, two, or more prior rewarded (R) or nonrewarded (N) events have occurred (see, e.g., Capaldi, 1985, 1994; Capaldi \& Miller, 1988; Capaldi \& Verry, 1981). Let SR1 represent the memory of a single prior R event, $\mathrm{SR} 2$ the memory of two successive prior events, and so on. Similarly, $\mathrm{S}^{\mathrm{N} 1}$ and $\mathrm{S}^{\mathrm{N} 2}$ represent the memories of one and two successive prior $\mathrm{N}$ events, respectively. It is clear that under any schedule of partial reward in a runway or similar apparatus, say a $50 \%$ irregular schedule, reward-produced memories cannot be less valid than exteroceptive cues and often are more valid. For example, under the $50 \%$ irregular schedule, no more than three $\mathrm{R}$ trials or $\mathrm{N}$ trials may occur in succession. Thus, while exteroceptive cues signal $\mathrm{R}$ on a $50 \%$ irregular basis, $\mathrm{S}^{\mathrm{N} 3}$ is a perfectly valid signal for $\mathrm{R}$ and $\mathrm{SR} 3$ is a perfectly valid signal for $\mathrm{N}$. The relative validities of other reward-produced memories, $\mathrm{SR} 1, \mathrm{~S}^{\mathrm{R}}, \mathrm{S}^{\mathrm{N}} 1$ and $\mathrm{SN}^{\mathrm{N}}$, would depend upon how the $50 \%$ irregular schedule is constructed in other respects (see Capaldi, 1994).

The purpose of the four investigations reported here was to provide experimental data for the suggestion that reward-produced memories might elicit expectancies, such as the expectancy of reward, $E_{R}$, and the expectancy of nonreward, $\mathrm{E}_{\mathrm{N}}$. The present hypothesis, that reward-produced memories and expectancies may work in tandem, was tested employing extinction. It is clear that stimuli characteristic of nonreward, such as $\mathrm{SN}^{\mathrm{N}}$ and $\mathrm{E}_{\mathrm{N}}$, occur in extinction. According to one view (e.g., Capaldi, 1966, 1967), resistance to extinction will be increased if stimuli such as $\mathrm{S}^{N 1}, \mathrm{SN}^{\mathrm{N}}$, etc., enter into an excitatory association with the instrumental response (IR) 
in acquisition. That is, if such cues are introduced in extinction for the first time, they will produce considerable generalization decrement, thereby promoting rapid extinction. According to another view (e.g., Amsel, 1992), resistance to extinction will be increased if stimuli associated with the expectancy of $\mathrm{N}$ enter into an excitatory association with IR in acquisition. Both views of extinction are accepted here.

Below are shown four different associative structures which are of particular concern in this report. Each of the first three structures is capable of producing increased resistance to extinction relative to the fourth. Structure 4 shows that $S^{R}$ elicits $E_{R}$, a Pavlovian relationship, and both $S R$ and $E_{R}$ elicit IR, an instrumental relationship. Structure 4 would arise under a schedule of consistent reinforcement, that is, $\mathrm{R}$ would be both remembered and expected on each trial and both $S^{R 1}$ and $E_{R}$ would occur on $R$ trials, and so both would enter into an excitatory relationship with IR. Structure 2 , in which $\mathrm{S}^{\mathrm{N} I}$ elicits $E_{R}$ and both elicit IR, would arise under a schedule of partial reward in which each $\mathrm{N}$ trial was followed by an R trial such as a schedule in which $\mathrm{R}$ and $\mathrm{N}$ trials alternated. Structure 3 , a relatively common structure, would arise under an irregular schedule of partial reward in which $\mathrm{N}$ trials were followed by $\mathrm{N}$ trials as well as by $R$ trials. It is Structure 1, which can arise only under unusual conditions, which is of major concern in the first three investigations reported here, inasmuch as it is being compared with other structures, for example, Structure 4. Structure 2 was examined in Experiment 4.

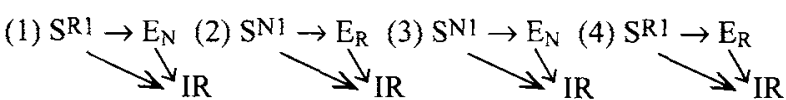

Two aspects of Structure 1 are worthy of attention. First, it produces increased resistance to extinction via an $E_{N} \rightarrow$ IR excitatory association and does not involve an $\mathrm{S}^{\mathrm{N}} \rightarrow$ IR excitatory association. Second, $\mathrm{E}_{\mathrm{N}}$ is elicited by $\mathrm{S}^{\mathrm{R} 1}$ and not by some exteroceptive cue. It follows that if results attributable to Structure $1 \mathrm{can}$ be obtained, support for the view that reward-produced memories can elicit expectancies will have been provided.

Experiment 1, conducted in three phases, employed a partial-reward schedule, in which an $\mathrm{R}$ trial was followed about $30 \mathrm{sec}$ later by an $\mathrm{N}$ trial, and a consistent reward schedule, in which an $\mathrm{R}$ trial was followed $30 \mathrm{sec}$ later by another $R$ trial. In designing Experiment 1, we assumed the following. An RN schedule given by itself would not produce increased resistance to extinction. However, if a shift occurred to the RR schedule following substantial training under the $\mathrm{RN}$ schedule, resistance to extinction would be increased. Our reasoning was as follows. Under the $\mathrm{RN}$ schedule, $\mathrm{S}^{\mathrm{R} 1}$ stored on Trial 1 would be retrieved on Trial 2 where it would come to elicit $E_{N}$. On Trial 2, an N trial, $S R 1$ and $E_{N}$ would enter into an inhibitory relationship with IR, producing slow running. When the shift occurred to the RR schedule, $S^{R I}$ would continue to elicit $E_{N}$ on Trial 2 for some number of shift trials. On such shift trials, $E_{N}$ would enter into an excitatory relationship with IR, because now $E_{N}$ occurs on an $R$ trial. In short, a shift from RN to RR would produce the associative structure shown under Structure 1.

In Experiment 1, two groups were employed, Group RN-RR and Group RR-RR. Following substantial training under the RN schedule, Group RN-RR was shifted to the RR schedule. Group RR-RR was trained under the RR schedule in both phases. Both groups were subsequently extinguished.

\section{EXPERIMENT 1}

\section{Method}

Subjects. The subjects were 10 experimentally naive male Holtzman albino rats purchased from the Harlan Co., Madison, Wisconsin. Their ages ranged from 90 to 95 days.

Apparatus. The apparatus in Experiment 1 was a straight gray runway, $197.10 \mathrm{~cm}$ long, $10.1 \mathrm{~cm}$ wide, and enclosed by $13.85-\mathrm{cm}$ sides; it was covered by a wire-mesh top on a hinged frame. The startbox and goalbox were 20.8 and $29.7 \mathrm{~cm}$ long, respectively, and were closed off by metal guillotine doors. Raising the startbox door started a completely silent 0.01 -sec digital clock, which was stopped when a photobeam, located $158.13 \mathrm{~cm}$ beyond the startbox door and $7.50 \mathrm{~cm}$ in front of the goal cup, was broken by the rat. Food (.045-g Noyes pellets) could be placed in the goal cup, which measured $4.00 \mathrm{~cm}$ in diameter and $1.50 \mathrm{~cm}$ in depth. When the photobeam was interrupted, an aluminum guillotine door was lowered, confining the rat to the goalbox.

Pretraining. On arrival at the laboratory, all rats were caged individually and given ad-lib food and water for 20 days. They were then placed on a deprivation diet consisting of $18 \mathrm{~g}$ of Purina Lab Chow each day. On Days 7-10 of deprivation, each rat was handled for about $1 \mathrm{~min}$. On Days 11-14, alley exploration was permitted for about $3 \mathrm{~min}$. On these days, $6.045 \mathrm{~g}$ Noyes pellets were scattered in the alley. At about the $11 / 2$-min mark of alley exploration each day, the guillotine doors were lowered and raised to habituate the rat to these events. The rats were fed their daily ration about $10 \mathrm{~min}$ after being returned to the home cage in all phases of the experiment.

Experimental training. Experimental training, which consisted of three phases (acquisition, transfer, and extinction), began on Day 15. A trial began with placement of the rat in the startbox. The start door was opened about $3 \mathrm{sec}$ later. A trial always terminated with the rat in the goalbox. If the rat did not enter the goalbox within $30 \mathrm{sec}$, it was picked up and placed there by the experimenter. Trials terminated either in food reward (R), $10.045-\mathrm{g}$ food pellets, or nonreward (N), $15 \mathrm{sec}$ confinement in the unbaited goalbox. All 10 rats were taken into the experimental room together. Each rat was placed in a separate cage in a rack. All rats received two trials in fairly rapid succession followed about $20 \mathrm{~min}$ later by two more trials in fairly rapid succession. This long interval was maintained by giving each rat its first series of two trials before any rat received its second series of two trials. The interval elapsing between the first and second trial was about $20 \mathrm{sec}$. The order of running the 10 rats was changed daily. There were two groups in Experiment 1. Group RR-RR received all $\mathrm{R}$ trials in training. On each of the first 11 days of experimental training, Group RN-RR was trained as follows: The first of its two trials of each pair terminated in $\mathrm{R}$, the second in N. Day 12 of experimental training was the first of a transfer phase in which Group RNRR was now trained identically to Group RR-RR. There were 5 days in transfer.

Extinction occurred on Days 17 and 18 of experimental training. Extinction occurred exactly as did acquisition and transfer, 
except that all trials terminated in N. Thus, on Days 17 and 18, each rat received two $\mathrm{N}$ trials in fairly rapid succession, followed about $20 \mathrm{~min}$ later by two more $\mathrm{N}$ trials in fairly rapid succession.

\section{Results}

Group RR-RR ran about as rapidly on Trial 1 as on Trial 2 of its RR sequence. However, as acquisition training progressed, Group RN-RR acquired the tendency to run more slowly on Trial 2, N, than on Trial 1, R. For example, on the last day of acquisition, Group RR-RR's speeds were 92.77 and $86.32 \mathrm{~cm} / \mathrm{sec}$, respectively. Those of Group RN-RR were 75.89 and $16.86 \mathrm{~cm} / \mathrm{sec}$, respectively. In acquisition, the following differences in speed of running were significant: groups $[F(1,8)=21.89, p<$ $.01]$, groups $\times$ trials $[F(1,8)=22.24, p<.01]$, and groups $\times$ trials $\times$ days $[F(1,80)=5.26, p<.01]$. These statistical findings indicated that Group RN-RR ran more slowly than Group RR-RR in the acquisition phase, that this difference was bigger on Trial 2 than on Trial 1 , and that the difference in running speed developed over days.

The most noteworthy result over the 5 days of transfer was that Group RN-RR showed an increase in running speed. By Day 5 of transfer, the speeds of Group RN-RR were 83.27 and $71.92 \mathrm{~cm} / \mathrm{sec}$ on Trials 1 and 2, respectively. The Trial 1 and Trial 2 speeds of Group RR-RR were 93.53 and $86.51 \mathrm{~cm} / \mathrm{sec}$, respectively. In transfer, the following differences were significant: groups $[F(1,8)=18.13, p<.01]$, groups $\times$ trials $[F(1,8)=$ $18.04, p<.01]$, and groups $\times$ trials $\times$ days $[F(4,32)=$ $3.73, p<.05]$. The significant triple interaction indicates that the speeds of Group RN-RR on Trial 2 increased substantially over the 5 days of transfer. For example, subsequent Newman-Keuls tests indicated that on Trial 2 Group RN ran faster on Day 5 than on Day 1 of transfer $(p<.01)$.

Figure 1 shows running speeds of Group RN-RR and Group RR-RR on Trials 1 and 2 on Day 5 of transfer (T) and on each of the 2 days of extinction. As may be seen, despite the fact that Group RN-RR ran more slowly than Group RR-RR on Day 5 of transfer, over the 2 days of extinction Group RN-RR ran more rapidly than Group RR-RR, a difference that was significant $[F(1,8)=6.76$, $p<.05$ ]. Running was slower on Trial 2 than on Trial 1 $[F(1,8)=48.81, p<.01]$, but this difference did not interact with groups $(F<1)$.

\section{Discussion}

In general terms, the hypothesis tested in Experiment 1 was that memories of prior reward events might acquire the capacity to elicit expectancies of reward events. Moreover, if expectancies occurred on rewarded trials, they might acquire the capacity to elicit IR. The hypothesis that memories and expectancies might work in tandem was confirmed in Experiment 1, with Group RN-RR showing greater resistance to extinction than Group RRRR. Specifically, it was assumed that under the RN schedule, $S^{R}$ came to elicit $E_{N}$. Subsequently, when the
TRIAL 1

TRIAL 2

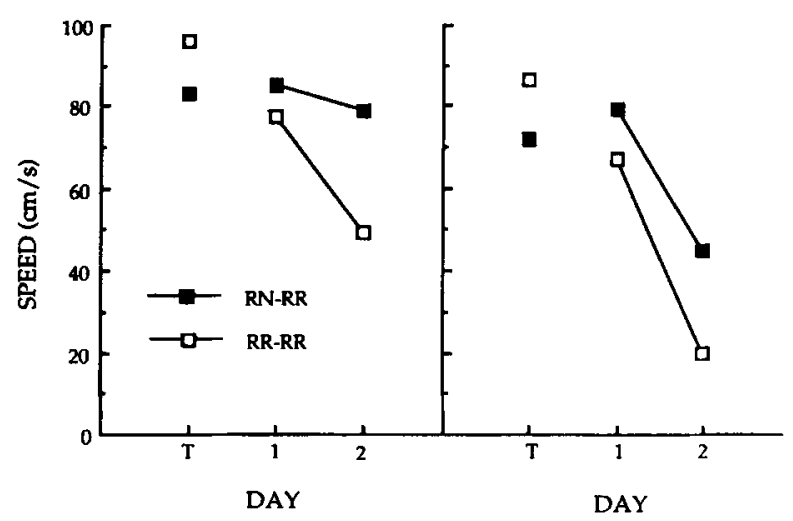

Figure 1. Running speeds (cm/sec) of Group RN-RR and Group RR-RR on Trial 1 (left panel) and on Trial 2 (right panel) on Day 5 of transfer $(T)$ and on each of the 2 days of extinction in Experiment 1.

shift occurred to the RR schedule, $E_{N}$ was elicited by $S R$ on Trial 2 of the RR schedule, an R trial, and so $E_{N}$ came to elicit IR. In Group RR-RR, $E_{N}$ was not conditioned to IR. Since $E_{N}$ occurred in extinction for both groups, Group RN-RR suffered less generalization decrement than Group RR-RR and thus showed the greater resistance to extinction.

While the findings obtained in Experiment 1 are consistent with the tandem hypothesis, viable alternative interpretations of those findings suggest themselves, two of which were tested in Experiment 2. For one, despite extensive prior evidence indicating that schedules such as RN fail to increase resistance to extinction (see, e.g., Capaldi, 1967, 1994), this may have been the case in Experiment 1 . Second, Group RN-RR was trained under two different schedules, while Group RR-RR was trained under only one. Perhaps training under two different schedules is sufficient in itself to increase resistance to extinction.

\section{EXPERIMENT 2}

Experiment 2 employed three groups. One was trained RN-RR, as in Experiment 1, one was trained RR-RN, and one was trained RN only (Group RN-RN). If two sequences in themselves elevate resistance to extinction, Group RR-RN should be as resistant to extinction as Group RN-RR and more resistant to extinction than Group RN-RN. If the RN sequence produces greater resistance to extinction than the RR sequence, Group RNRN should be as resistant to extinction as Group RN$R R$. The tandem hypothesis predicts, however, that Group RN-RR should show greater resistance to extinction than Groups RR-RN and RN-RN. This is because, in transfer in Group RN-RR, $E_{N}$ should occur on Trial 2 of the $R R$ sequence, an $R$ trial, and so $E_{N}$ should acquire the capacity to elicit the IR, something which should not be true for Groups RN-RN and RR-RN. 


\section{Method}

Subjects. The subjects were 15 rats of the same description as those employed in Experiment 1.

Apparatus. The same apparatus as that employed in Experiment 1 was used in Experiment 2.

Preliminary training. Preliminary training was as in Experiment 1.

Experimental training. Experimental training was exactly as in Experiment 1, except for the following differences: There were three groups of 5 rats each. All rats were trained for 25 days prior to extinction. On each of the 25 days, Group RN-RN was trained $R N$, that is, the first trial was R, the second N. Groups RR-RN and RN-RR received the RR schedule for 5 days and the RN schedule for 20 days. The difference was this: Group RR-RN received the RR sequence on the initial 5 days before being transferred to $R N$ (Transfer 1). Group RN-RR received the RR schedule on the last 5 days, after being transferred from the RN schedule to the RR schedule (Transfer 2). Because of the greater number of rats in Experiment 2 , the interval between the first and second series of two trials was longer, about 30 min. Extinction began on Day 26 and lasted for 4 days.

\section{Results}

Over the initial 5 days of experimental training, Group RR-RN ran faster than Groups RN-RR and RN-RN $[F(2,11)=22.58, p<.001]$. On Day 5, for example, speeds on Trials 1 and 2 , respectively, were 55.89 and $70.9 \mathrm{~cm} / \mathrm{sec}$ for Group RR-RN, 23.3 and $18.3 \mathrm{~cm} / \mathrm{sec}$ for Group RN-RR, and 24.1 and $20.8 \mathrm{~cm} / \mathrm{sec}$ for Group RN-RN.

Over Days 1-20 of Transfer 1, in which all groups were trained $\mathrm{RN}$, speeds on $\mathrm{R}$ trials exceeded those on $\mathrm{N}$ trials and differences between the groups became negligible $[F(2,11)=2.30, p>.05]$. On Day 20 of Transfer 1 , for example, speeds on $\mathrm{R}$ and $\mathrm{N}$ trials, respectively, were 81.8 and $38.9 \mathrm{~cm} / \mathrm{sec}$ for Group RR-RN, 76.7 and $35.4 \mathrm{~cm} / \mathrm{sec}$ for Group RN-RR, and 73.8 and $36.0 \mathrm{~cm} / \mathrm{sec}$ for Group RN-RN.

Over Days 1-5 of Transfer 2, Group RN-RR, now trained RR, increased its speeds on the second trial of the sequence. On these days, while difference between the groups was not significant $[F(2,11)=1.90, p>.05]$, the group $\times$ trial $\times$ day interaction was significant $[F(8,44)=$ $4.54, p<.001]$. Over the 5 days of Transfer 2 , running speeds on Trials 1 and 2 were, respectively, 76.11 and $56.32 \mathrm{~cm} / \mathrm{sec}$ for Group RN-RR, 73.91 and $28.24 \mathrm{~cm} / \mathrm{sec}$ for Group RR-RN, and 75.62 and $23.95 \mathrm{~cm} / \mathrm{sec}$ for Group RN-RN.

Figure 2 shows running speeds of each of the three groups on Trials 1 and 2 on Day 5 of Transfer 2 and on each of the 4 days of extinction. As may be seen on Day 5 of Transfer 2, the three groups ran about at the same speed on Trial 1 of their respective sequences. However, by Day 5 of Transfer 2, Group RN-RR ran almost as rapidly on Trial 2 as on Trial 1 , while the two other groups ran much more rapidly on Trial 1 than on Trial 2. Over the 4 days of extinction, Group RN-RR ran more rapidly than either Group RR-RN or Group RN$\mathrm{RN}$ on Trials 1 and 2, the latter two groups not differing from each other. In extinction the following differences were significant: groups $[F(2,11)=6.80, p<.05]$, groups $\times$ trials $[F(2,11)=7.38, p<.01]$, and groups $\times$ trials $\times$
TRIAL 1

TRIAL 2

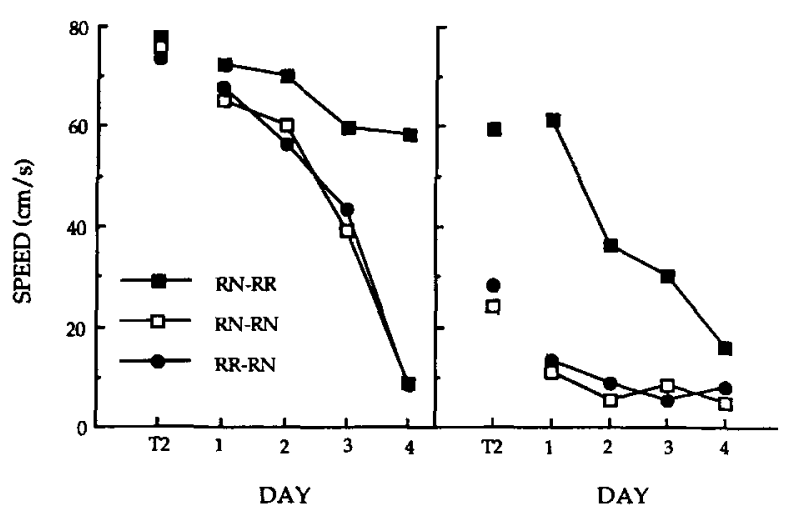

Figure 2. Running speeds $(\mathrm{cm} / \mathrm{sec})$ of each of the three groups on Trial 1 (left panel) and Trial 2 (right panel) on Day 5 of Transfer 2 and on each of the 4 days of extinction in Experiment 2.

days $[F(6,33)=8.56, p<.001]$. Subsequent NewmanKeuls tests based upon the significant triple interaction revealed the following. On each of the first 3 days of extinction, Group RN-RR ran significantly faster on Trial 2 of extinction than either Group RR-RN or Group RN$\mathrm{RN}(p \mathrm{~s}<.05)$. On Trial 1, Group RN-RR differed from Groups RR-RN and RN-RN on Days 3 and 4 ( $p s<.05$ ). No other difference between the groups was significant.

\section{Discussion}

In extinction, Group RN-RR ran faster than Groups RR-RN and RN-RN on Trial 1 and on Trial 2 of the NN extinction schedule. Prior to extinction, Group RN-RR ran faster on Trial 2 of the day than either Group RR-RN or Group RN-RN. Thus, that Group RN-RR ran fastest on Trial 2 of extinction may merely reflect acquisition differences. However, in acquisition, all three groups ran equally rapidly on Trial 1 . Thus, the finding that Group RN-RR ran significantly faster than Groups RRRN and RN-RN on Trial 1 of the NN sequence in extinction can be taken to indicate that that group was more resistant to extinction than were Groups RR-RN and RN-RN. Recall, too, that in Experiment 1, Group RN$R R$, while slower than Group RR-RR on Day 5 of transfer, nevertheless ran faster than Group RR-RR in extinction. None of the meaningful extinction differences obtained in Experiments 1 and 2 can be attributed to differences in acquisition. The present findings are consistent with the hypothesis that the memories of prior reward events may elicit the expectancies of reward events.

The Experiment 2 findings are inconsistent with the two alternative interpretations of the findings obtained in Experiment 1. They are inconsistent with the hypothesis that the RN schedule in itself elevates resistance to extinction relative to the RR schedule. They are inconsistent with the hypothesis that the RN and RR schedule, if given together in either order, produce greater resistance to extinction than either schedule alone. Thus, the 
findings from both Experiment 1 and Experiment 2 indicate that a shift from an RN schedule to an RR schedule increases resistance to extinction, as suggested by the tandem hypothesis.

It should be noted that in the absence of an expectancy assumption it would be difficult to explain why a shift from $R N$ to $R R$ produces increased resistance to extinction. Assume, for example, that under the RN schedule, $\mathrm{SR}$, by occurring on Trial 2, an $\mathrm{N}$ trial, establishes an inhibitory relationship with IR, thereby producing slow running, but that the animal has no expectancy of $N$ on Trial 2. On the shift to RR, $S^{R}$ would now occur on an $R$ trial and so establish an excitatory relationship with IR. But an excitatory relationship between $S R$ and IR is also established under the RR schedule. Why, then, would a shift from RN to RR elevate resistance to extinction relative to $R R$ ? We conclude that, in the absence of an expectancy assumption, it is difficult to explain why the shift from an $\mathrm{RN}$ to an $R R$ schedule elevates resistance to extinction relative to an RR schedule.

According to the tandem hypothesis, in groups shifted from RN to RR, $S R$ came to elicit $E_{N}$. Experiment 3 tested an alternative to the tandem hypothesis. According to that alternative, $E_{N}$ was conditioned to IR on Trial 2 of the RR schedule as the tandem hypothesis suggests. However, according to the alternative view, $\mathrm{E}_{\mathrm{N}}$ was not elicited by SR but by Trial 2 related stimuli such as the time elapsing from the completion of Trial 1 to the onset of Trial 2. That alternative view assumes, reasonably enough, that rats are able to discriminate between Trial 1 and Trial 2 of the day on some basis. According to this alternative view, Trial 1 cues would come to elicit $E_{R}$, while Trial 2 cues would come to elicit $E_{N}$. On being shifted to RR, the Trial 2 cues would tend to elicit $E_{N}$ on Trial 2 of that schedule, resulting in the $E_{N} \rightarrow I R$ association which would result in increased resistance to extinction.

It should be noted that the tandem hypothesis recognizes that expectancies may be elicited by stimuli other than reward-produced memories. Indeed, even in the specific case under examination, the shift from $R N$ to $\mathrm{RR}$, it seems possible that in addition to being elicited by $S R, E_{N}$ may be elicited by other cues as well. However, available evidence indicates that even when reward-produced memories, such as $\mathrm{SR} 1$ and $\mathrm{S}^{\mathrm{N}} 1$, have occurred in compound with highly discriminable exteroceptive cues, such as brightness cues or a flashing light, they have nevertheless acquired control over responding; that is, the reward-produced memories were not completely overshadowed (e.g., Haddad et al., 1981; Haggbloom, 1981). Given these findings for brightness cues and flashing lights, it seems unlikely that the reward-produced memories would be completely overshadowed by the seemingly less salient trial-related stimuli. In Experiment 3, we attempted to determine if trial-related stimuli would completely deprive $S^{R}$ of control over $\mathrm{E}_{\mathrm{N}}$.

\section{EXPERIMENT 3}

Experiment 3 contained two groups. Group NN received the two-trial schedule $\mathrm{NN}$ and a single $\mathrm{R}$ trial. Group RN was trained similarly, except that it received the two-trial schedule RN and a single $\mathrm{N}$ trial. Eventually both groups were shifted to the RR schedule, and subsequent to this both groups were extinguished.

On Trial 2, both groups were nonrewarded prior to shift. Assume that, in both groups in the initial stage of training, $E_{N}$ came to be elicited by Trial 2 stimuli, and only by Trial 2 stimuli. In that event, $E_{N}$ would be conditioned equally to the IR in both groups on Trial 2 of the $R R$ schedule in transfer. Thus, the two groups should not differ in extinction. The tandem hypothesis, however, suggests the following: In the original learning phase, regardless of the role played by Trial 2 stimuli, $\mathrm{E}_{\mathrm{N}}$ would be elicited by $S^{R}$ in Group $R N$ and by $S^{N}$ in Group NN. In shift, under the $R R$ sequence, $E_{N}$ would be elicited on Trial 2 by $S R$ in both groups. $S R$ is a directly conditioned stimulus for $E_{N}$ in Group $R N$ and a generalized stimulus in Group NN. It follows that on Trial 2 of the RR schedule, $E_{N}$ would be elicited more strongly and perhaps more reliably in Group RN than in Group NN. On this basis, we could expect $\mathrm{E}_{\mathrm{N}}$ to become more strongly associated with IR in Group RN than in Group NN. Thus, Group $\mathrm{RN}$ should show the greater resistance to extinction.

\section{Method}

Subjects. The subjects were 10 rats of the same description as those employed in Experiment 1.

Apparatus. The apparatus was the same as that employed in Experiments 1 and 2.

Preliminary training. Preliminary training was as in Experiment 1 , except for the following differences. The rats were given ad-lib food and water for only 15 days. The rats were handled on Days 8-10 of deprivation for about $20 \mathrm{~min}$ in groups of 5 . On Days $11-15$ of alley exploration, ten .045 -g Noyes pellets were scattered in the alley.

Experimental training. Experimental training was similar to that employed in Experiment 1, except for the following differences. There were 20 days of acquisition training followed by 6 days of transfer prior to extinction. On each day of acquisition, Group RN received an RN schedule and a single N trial, separated by about a $15-\mathrm{min}$ interval. The RN schedule occurred prior to the single $\mathrm{N}$ trial on odd days and following the single $\mathrm{N}$ trial on even days. Group NN was trained similarly, except that it received an $\mathrm{NN}$ schedule and a single $\mathrm{R}$ trial. In transfer, both groups were trained under the RR schedule, as in Experiments 1 and 2, and there were 2 days of extinction, as in Experiment 1.

\section{Results}

Over the 20 days of acquisition, both groups ran rapidly on Trial 1, developing the tendency to run slowly on Trial 2. Analysis of variance indicates that while Trial 2 speeds were slower than Trial 1 speeds $[F(1,8)=19.25$, $p<.01]$, no differences involving groups were significant. On the last day of acquisition, Group RN speeds on Trials 1 and 2 were 72.85 and $17.81 \mathrm{~cm} / \mathrm{sec}$, respectively, while those of Group NN were 70.89 and $17.46 \mathrm{~cm} / \mathrm{sec}$, respectively. 
TRIAL 1

TRIAL 2

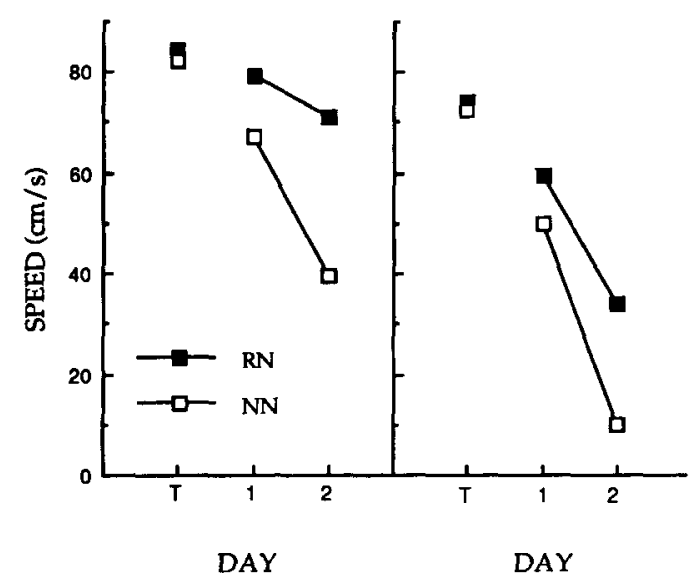

Figure 3. Running speeds of Group RN and Group NN on Trial 1 (left panel) and on Trial 2 (right panel) on Day 6 of transfer (T) and on each of the 2 days of extinction in Experiment 3.

Over the 6 days of transfer under the RR schedule, the speeds of both groups improved substantially. On the last day of transfer, for example, Group $\mathrm{RN}$ speeds were 84.52 and $73.18 \mathrm{~cm} / \mathrm{sec}$ on Trials 1 and 2, respectively, while those of Group NN were 82.91 and $72.49 \mathrm{~cm} / \mathrm{sec}$, respectively. In transfer, no difference involving group was significant.

Figure 3 shows the running speeds for Group $\mathrm{RN}$ and Group NN on Trials 1 and 2 on Day 6 of transfer (T) and on each of the 2 days of extinction. In extinction, Group $\mathrm{RN}$ ran more rapidly than Group $\mathrm{NN}$, a difference that was significant $[F(1,8)=12.32, p<.05]$. Running was slower on Trial 2 than on Trial $1[F(1,8)=29.79, p<.01]$.

\section{Discussion}

In Experiment 3, it was found that Group RN showed greater resistance to extinction than Group NN. This finding, as shown earlier, is consistent with the view that $S^{R}$ elicited $E_{N}$, that is, that reward-produced memoiies can acquire control over expectancies. At the same time, the results obtained in Experiment 3 cannot be taken to suggest that Trial 2-related cues did not come to elicit $E_{N}$ in both groups. If they did, and they may have, it may be concluded that in the shift phase the stimulus compound $\mathrm{SR}_{\mathrm{R}}$ and Trial 2-related cues elicited $\mathrm{E}_{\mathrm{N}}$ more strongly in Group RN than in Group NN.

Given the reward schedule employed in Experiment 3, $E_{N}$ could have been, and probably was, (1) elicited by stimuli other than the reward-produced memories in both groups, and (2) occurred on R trials in both groups. In these cases, $E_{N}$ would have acquired control over responding in both groups. As a major case in point, partial reward occurred on Trial 1 in both groups. Thus, on Trial 1, $\mathrm{E}_{\mathrm{N}}$ would have occurred on $\mathrm{R}$ trials in both groups. But the control over responding that $E_{N}$ may have acquired in this particular case was not different in the two groups and could not explain why the groups differed in extinction.

As indicated, previous investigations have shown that $\mathrm{S}^{\mathrm{R}}$ and $\mathrm{S}^{\mathrm{N}}$ are not easily overshadowed by exteroceptive cues (e.g., Haddad et al., 1981; Haggbloom, 1981). The results obtained in Experiment 3 indicate that trialrelated stimuli, whatever their role may have been, did not completely deprive $S R$ of the capacity to elicit $E_{N}$.

\section{EXPERIMENT 4}

Another alternative to the tandem hypothesis is one involving expectancy alone. Applied to the present investigations, that view would suggest that under, for example, the RN schedule, the following occurred: On the basis of the Trial 1 events and prior to Trial 2, the animal instructs itself that nonreward will occur on Trial 2, and it is this instruction which is retained in the retention interval separating Trial 1 and Trial 2 (see, e.g., Chatlosh \& Wasserman, 1992; Wasserman, 1986). The difference between the expectancy-alone hypothesis and the present one is as follows: The expectancy-alone hypothesis suggests that on Trial 2 of, for example, the RN schedule, only $E_{N}$ occurs, while the tandem hypothesis suggests that on Trial 2 of the RN schedule $S^{R}$ elicits $E_{N}$. Both views would suggest that when a shift occurs from $\mathrm{RN}$ to RR, $\mathrm{E}_{\mathrm{N}}$ would occur on Trial 2 of the RR schedule, and so both views would predict that the shift from RN to RR would produce increased resistance to extinction. However, while both views can explain the findings in Experiments 1 and 2, only the tandem hypothesis appears able to explain those obtained in Experiment 3.

In Experiment 3, both Group RN and Group NN could instruct themselves in the original learning phase that $\mathrm{N}$ was to occur on Trial 2. Moreover, both groups experienced the same RR schedule in the shift phase, and so in both groups $E_{N}$ had the same opportunity to become conditioned to IR. This analysis, as may be seen, provides no basis for expecting Group RN to show greater resistance to extinction than Group NN, as was found in Experiment 3. While the results obtained in Experiment 3 do not seem consistent with the expectancyalone view, they do not demonstrate as directly as may be desired that memories of reward events occurred on Trial 2 of the schedules employed here.

In Experiment 4, we attempted to demonstrate rather directly that memories of prior reward events may not be discarded when correct expectancies of reward events on the next trial can be formed prior to the occurrence of the next trial. In Experiments 1, 2, and 3, increased resistance to extinction was attributed to the behavioral control exercised by $\mathrm{E}_{\mathrm{N}}$. In Experiment 4, increased resistance to extinction, if it occurred, could be attributed only to the behavioral control exercised by $\mathrm{S}^{\mathrm{N}}$. In $\mathrm{Ex}-$ periment 4 , extinction followed original acquisition, that is, there was no shift phase.

Phase 1 of Experiment 4 was conducted like Phase 1 of Experiment 3, except that Group NR received the 
two-trial schedule NR and a single R trial, while Group $R R$ received the two-trial schedule $R R$ and a single $N$ trial. In Experiment 4, both groups received 100\% reward on Trial 2. Following this training, both groups were extinguished. According to the expectancy-only hypothesis, on Trial 2 both groups would (1) anticipate $\mathrm{R}$ and (2) forget the Trial 1 reward outcome. Thus, according to the expectancy-alone view, both groups would traverse the runway on Trial 2 in the presence of $E_{R}$, and so should not differ in extinction. According to the tandem hypothesis, Group NR should show greater resistance to extinction than Group RR for the following reason: On Trial 2, Group NR would respond in the presence of $S^{N}$ and $E_{R}\left(S^{N} \rightarrow E_{R}\right)$ while Group $R R$ would respond in the presence of $S^{R}$ and $E_{R}\left(S^{R} \rightarrow E_{R}\right)$. Thus, $\mathrm{S}^{\mathrm{N}}$ would be conditioned to the instrumental response in Group NR but not in Group RR, and so Group NR should show the greater resistance to extinction. Note that in both groups, because of $50 \%$ irregular partial reward on Trial 1 of their schedules, $\mathrm{E}_{\mathrm{N}}$ would acquire control over responding. However, only in Group NR would SN acquire control over responding on Trial 2. Note, too, that according to the present hypothesis, in both groups $\mathrm{R}$ on Trial 2 is as validly signaled by trialrelated cues as by reward-produced memories. Thus, if Group NR showed greater resistance to extinction than Group RR, additional evidence would have been provided that reward-produced memories can be effective cues even when they occur in compound with equally valid trial-related stimuli. Finally, note that the associative structure formed in Group NR would be that shown under Structure 2, described in the introductory section.

\section{Method}

Subjects. The subjects were 10 rats of the same description as those employed in Experiment 1.

Apparatus. The apparatus was the same as that employed in Experiment 1.

Preliminary training. Preliminary training was identical to that employed in Experiment 3.

Experimental training. Experimental training was identical to that employed in Experiment 3, except for the following differences. Group RR received an RR schedule and a single $\mathrm{N}$ trial, while Group NR received an NR schedule and a single R trial. Following 20 days of the above training, the rats received 3 days of extinction training.

\section{Results}

Figure 4 shows running speeds for Groups NR and RR on the last day of acquisition (A) and on each of the 3 days of extinction for Trials 1 and 2 . On the last day of acquisition, as on each of the preceding days, the speeds of the two groups were practically identical $(F<1)$. Speeds in acquisition were slightly faster on Trial $2(100 \%$ reward) than on Trial 1 (50\% irregular reward), but this difference was not significant $(F<1)$. In extinction, Group NR ran faster than Group RR on Trial 1 and on Trial 2, a difference which was highly significant $[F(1,8)=$ $18.55, p<.01]$. Running speeds were faster on Trial 1 than on Trial $2[F(1,8)=6.09, p<.05]$.

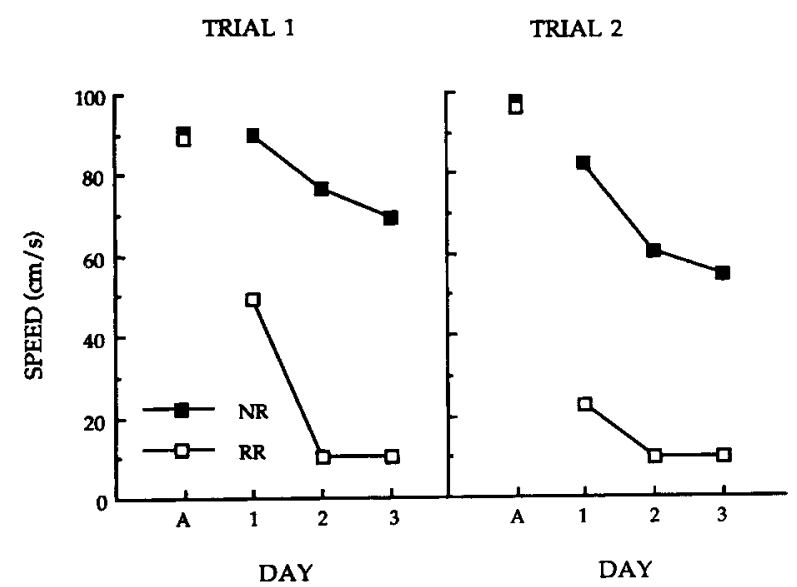

Figure 4. Running speed of Groups NR and RR on the last day of acquisition (A) and on each of the 3 days of extinction for Trial 1 and Trial 2 in Experiment 4.

\section{Discussion}

Group NR showed greater resistance to extinction than Group RR. This finding supports the tandem hypothesis. The finding obtained in Experiment 4 is consistent with a variety of earlier findings which suggest that on current trials the animal may remember one or more reward events which occurred on prior trials, while simultaneously anticipating one or more reward events which are to occur on subsequent trials (see, e.g., Capaldi, 1985, 1993; Capaldi \& Verry, 1981). While the findings obtained in Experiment 4 support the tandem hypothesis, they do not suggest rejection of all aspects of the expectancy-alone hypothesis. That hypothesis suggests, of course, that on Trial 1 the animal may come to expect the Trial 2 reward event. As one example of this, we suspect that when $\mathrm{R}$ occurs on Trial 1 under an RN sequence, the animal may well anticipate that $\mathrm{N}$ will occur on Trial 2 prior to receiving Trial 2 (see, e.g., Capaldi, 1994). However, as is perhaps clear, we believe that on Trial 2 the animal also remembers R. Applied to the NR schedule used in Experiment 4, this view suggests that on the $\mathrm{N}$ trial the animal may well anticipate the subsequent $R$ event in advance of the $R$ trial, but when Trial 2 occurs, $S^{N}$ stored on Trial 1 is retrieved and $\mathrm{S}^{\mathrm{N}}$ elicits the IR.

On Trial 2, in both Group NR and Group RR, R was as validly predicted by Trial 2-related stimuli as by retrospective memories of goal events. If those Trial 2related stimuli had completely overshadowed the retrospective memories, Groups NR and RR would not have differed in extinction. Thus, we may assume that in Group NR, $S^{N}$ acquired some capacity to elicit responding in Group NR despite occurring in compound with Trial 2related cues. Of course, the distinctive Trial 2 stimuli may have reduced the control acquired by $\mathrm{SN}$ in Group NR. In any event, Experiment 3 demonstrated that $E_{N}$ was not completely overshadowed by trial-related stimuli, and Experiment 4 demonstrated the same for $\mathrm{S}^{\mathrm{N}}$. 


\section{GENERAL DISCUSSION}

Expectancies of reward events such as $E_{R}$ and $E_{N}$ are widely assumed to be established on the basis of Pavlovian conditioning. According to the tandem hypothesis, memories of prior reward events such as $\mathrm{S}^{\mathrm{R}}$ and $\mathrm{S}^{\mathrm{N}}$ may serve as conditioned stimuli for expectancies. The tandem hypothesis was supported by the results obtained in the four investigations reported here. It was found, in Experiments 1 and 2, that a shift from an RN to an RR schedule of reward produced increased resistance to extinction relative to an RR schedule, an RN schedule, or one in which a shift occurred from RR to RN. These findings were interpreted as indicating that, under the $\mathrm{RN}$ schedule, $\mathrm{SR}$ comes to elicit $\mathrm{E}_{\mathrm{N}}$, with both stimuli subsequently coming to elicit IR in transfer under the RR schedule. Under each of the other three schedules mentioned, RR alone, RN alone, or RR shifted to RN, $E_{N}$ does not come to elicit IR. In extinction, $E_{N}$ occurs. Thus, where $E_{N}$ has acquired the capacity to elicit IR, resistance to extinction will be increased.

Granting that a shift from an RN schedule to an RR schedule allows $E_{N}$ to acquire the capacity to elicit IR, it is possible that $E_{N}$ was elicited not by $S R$ but by stimuli associated with Trial 2. Experiment 3, by showing that a shift from RN and N to RR produced greater resistance to extinction than a shift from $N N$ and $R$ to $R R$, provided evidence that $S^{R}$ is part of the stimulus complex eliciting $E_{N}$. The results obtained in Experiment 3 do not preclude the possibility that Trial 2-related stimuli are part of the stimulus complex eliciting $\mathrm{E}_{\mathrm{N}}$, along with SR. In Experiment 4, it was found that a group trained NR and $R$ showed greater resistance to extinction than a group trained $R R$ and $N$. This finding indicates that rats traversed the runway on Trial 2 in the presence of both reward-produced memories and expectancies rather than expectancies alone. These findings indicate, as do those of Experiment 3, that when reward-produced memories occur in compound with trial-related stimuli, the former nevertheless acquire some capacity to elicit expectancies.

A shift from an RN schedule to an RR schedule is in general terms a shift from a schedule of partial reward $(\mathrm{P})$ to a schedule of consistent reward (C), or a $\mathrm{P}-\mathrm{C}$ shift. In prior investigations a shift from an irregular $P$ schedule to a $\mathrm{C}$ schedule failed to increase resistance to extinction (see, e.g., Sutherland, Mackintosh, \& Wolfe, 1965; Theios, 1962), while a shift from a $P$ schedule in which $\mathrm{R}$ and $\mathrm{N}$ trials alternated to $\mathrm{C}$ produced increased resistance to extinction (Campbell, Knouse, \& Wroten, 1970). Amsel (1992) has explained the former but not the latter findings in terms of frustration theory. However, both findings are consistent with the tandem hypothesis. Under the irregular schedule, as is perhaps clear from prior comments in the introductory section, $\mathrm{E}_{\mathrm{N}}$ would acquire the tendency to elicit IR prior to the shift to the $\mathrm{C}$ schedule (Structure 3 ). This would not be the case under the single-alternation schedule, since under that schedule $\mathrm{S}^{\mathrm{R}}$ elicits $\mathrm{E}_{\mathrm{N}}$ on $\mathrm{N}$ trials and $\mathrm{S}^{\mathrm{N}}$ elicits $E_{R}$ on $R$ trials. On the shift from single-alternation $P$ to the $C$ schedule, $S^{R}$ would elicit $E_{N}$ on $R$ trials. To sum up, on the shift from irregular $P$ to $C, E_{N}$ does not acquire a substantial increase in its capacity to elicit IR, whereas it does do so on the shift from single-alternation $\mathrm{P}$ to $\mathrm{C}$. Thus, both sets of findings are consistent with the tandem hypothesis.

As to the conditions under which reward-produced memories come to elicit expectancies, we consider below a general, rather than a specific, answer. In any situation in which the reward-produced memories are more valid than other stimuli compounded with them, the rewardproduced memories, we suggest, will acquire some control over expectancies. Where the reward-produced memories are merely as valid as other stimuli compounded with them, the memories may acquire some control over expectancies, since they appear to be relatively salient stimuli (e.g., Haddad et al., 1981; Haggbloom, 1981). Under what conditions are rewardproduced memories as valid as or more valid than various other stimuli? That is an empirical matter. A more or less extended discussion of these matters has been provided elsewhere (Capaldi, 1994). As that discussion indicates, the possibility that reward-produced memories may be relatively valid under a wide variety of conditions cannot be precluded on the basis of available information.

In a recent series of experiments, Haggbloom (1988), among other things, rewarded rats in the presence of an exteroceptive $\mathrm{S}-$ cue, for example, a tactile cue. This produced increased resistance to extinction relative to a variety of controls. I interpret Haggbloom's (1988) findings in the same terms as the shifts from singlealternation $\mathrm{P}$ to $\mathrm{C}$ or RN to RR; in the shift phase, an Scue gave rise to $E^{N}$, which became a signal for R. In Haggbloom's case, the $S-$ cue was an exteroceptive cue. In our investigations and in that of Campbell et al. (1970), the $\mathrm{S}-$ cue was an interoceptive cue, $\mathrm{SR}$. As this analysis shows, partial-reward schedules in which reward and nonreward may be predicted as in, for example, the RN schedule, may be conceptualized as discriminationlearning situations in which reward-produced memories serve as the discriminative cues. Of course, it has long been recognized that partial reward and discrimination learning may involve similar mechanisms to a considerable extent (see, e.g., Amsel, 1992; Capaldi, 1994).

\section{REFERENCES}

Amsel, A. (1958). The role of frustrative nonreward in noncontinuous reward situations. Psychological Bulletin, 55, 102-119.

AMSEL, A. (1992). Frustration theory: An analysis of dispositional learning and memory. New York: Cambridge University Press.

Campbell, P. E., Knouse, S. B., \& Wroten, J. D. (1970). Resistance to extinction in the rat following regular and irregular schedules of partial reward. Journal of Comparative \& Physiological Psychology, 72, 210-215.

Capald, E. J. (1966). Partial reinforcement: A hypothesis of sequential effects. Psychological Review, 73, 459-477. 
CAPALDI, E. J. (1967). A sequential hypothesis of instrumental learning. In K. W. Spence \& J. Spence (Eds.), The psychology of learning and motivation (Vol. 1, pp. 67-156). New York: Academic Press.

CAPAldi, E. J. (1985). Anticipation and remote associations: A configural approach. Journal of Experimental Psychology; Learning, Memory, \& Cognition, 11, 444-449.

CAPALDI, E. J. (1993). The basis of prospective memory in retrospective memory. Invited paper presented at the Sixty-fifth Annual Meeting of the Midwestern Psychological Association, Chicago.

CAPALDI, E. J. (1994). The sequential view: From rapidly fading stimulus traces to the organization of memory and the abstract concept of number. Psychonomic Bulletin \& Review, 1, 156-181.

CAPAldi, E. J., \& MiLleR, D. J. (1988). The rat's simultaneous anticipation of remote events and current events can be sustained by event memories alone. Animal Learning \& Behavior, 16, 1-7.

Capaldi, E. J., \& Verry, D. R. (1981). Serial anticipation learning in rats: Memory for multiple hedonic events and their order. Animal Learning \& Behavior, 9, 441-453.

Chatlosh, D. L., \& Wasserman, E. A. (1992). Memory and expectancy in delayed discrimination procedures. In I. Gormezano \& E. A. Wasserman (Eds.), Learning and memory: The behavioral and biological substrates (pp. 61-79). Hillsdale, NJ: Erlbaum.

Haddad, N. F., Walkenbach, J., Preston, M., \& Strong, R. (1981). Stimulus control in a simple instrumental task: The role of internal and external stimuli. Learning \& Motivation, 12, 509-520.
HAGGBLOOM, S. J. (1981). Blocking in successive differential conditioning: Prior acquisition of control by internal cues blocks the acquisition of control by brightness. Learning \& Motivation, 12, 485 508 .

HaGGBLoOM, S. J. (1988). The signal-generated partial reinforcement extinction effect. Journal of Experimental Psychology: Animal Behavior Processes, 14, 89-95.

Hilgard, E. R., \& MARQuis, D. G. (1940). Conditioning and learning. New York: Appleton-Century-Crofts.

Sutherland, N. S., Mackintosh, N. J., \& Wolfe, J. B. (1965). Extinction as a function of the order of partial and consistent reinforcement. Journal of Experimental Psychology, 69, 56-59.

THEIOS, J. (1962). The partial reinforcement effect sustained through blocks of continuous reinforcement. Journal of Experimental Psychology, 64, 1-6.

TolmaN, E. C. (1934). Theories of learning: Formulation of the theories and their adequacy in the light of data collected. In F. A. Moss (Ed.), Comparative psychology (pp. 367-408). New York: Prentice-Hall. WASSERMAN, E. A. (1986). Prospection and retrospection as processes of animal short-term memory. In D. F. Kendrick, M. E. Rilling, \& M. R. Denny (Eds.), Theories of animal memory (pp. 53-75). Hillsdale, NJ: Erlbaum.

(Manuscript received October 29, 1993; revision accepted for publication March 24, 1994.) 